\title{
CORRIGENDUM
}

\section{Destructive extraction of phospholipids from Escherichia coli membranes by}

graphene nanosheets

Yusong Tu, Min Lv, Peng Xiu, Tien Huynh, Meng Zhang, Matteo Castelli, Zengrong Liu, Qing Huang, Chunhai Fan,

Haiping Fang and Ruhong Zhou

Nature Nanotechnology 8, 594-601 (2013); published online 7 July 2013; corrected after print 26 November 2013.

In the version of this Article originally published, it was not made clear that the two Escherichia coli cells in the bottom left of Fig. 1a are from a different TEM image to the others. The figure and caption have now been corrected in the PDF and HTML versions of the Article. 